\title{
Energy density fluctuations in Early Universe
}

\author{
G.L. Guardo*, V. Greco*, and M. Ruggieri* \\ *Department of Physics and Astronomy, University of Catania, Catania, Italy \\ ${ }^{\dagger} I N F N$ - Laboratori Nazionali del Sud, Catania, Italy
}

\begin{abstract}
The primordial nucleosinthesys of the element can be influenced by the transitions of phase that take place after the Big Bang, such as the QCD transition. In order to study the effect of this phase transition, in this work we compute the time evolution of thermodynamical quantities of the early universe, focusing on temperature and energy density fluctuations, by solving the relevant equations of motion using as input the lattice QCD equation of state to describe the strongly interacting matter in the early universe plasma. We also study the effect of a primordial strong magnetic field by means of a phenomenological equation of state. Our results show that small inhomogeneities of strongly interacting matter in the early Universe are moderately damped during the crossover.
\end{abstract}

Keywords: Quark-gluon plasma, Early universe, Primordial magnetic fields.

PACS: $12.38 . \mathrm{Gc}, 12.38 . \mathrm{Mh}, 12.39 . \mathrm{Ba}, 98.80 . \mathrm{Bp}$

\section{INTRODUCTION}

The history of the early universe left an imprint on the currently observed cosmos. According to the Friedmann equations, the temperature $T$ of the early universe plasma decreased as $a^{-1}$ with $a$ the scale parameter, so that several phase transitions occurred during the early evolution of the universe, like the electroweak phase transition at $t \approx 10^{-10}$ $\sec$ (corresponding to $\mathrm{T} \approx 100 \mathrm{GeV}$ ) and the $\mathrm{QCD}$ phase transition at $\mathrm{t} \approx 10^{-5} \mathrm{sec}(\mathrm{T} \approx 150 \mathrm{MeV})$, then passing through the Big Bang Nucleosynthesis (BBN) at $t=1 \mathrm{sec}-3 \min (\mathrm{T}=0.1-1 \mathrm{MeV})$. This $\mathrm{BBN}$ plays a crucial role in constraining our views of the universe: it is essentially the only probe for the radiation dominated epoch during the range $\approx 1-10^{4}$ sec. Moreover, the aforementioned phase transitions could strongly affect the BBN [1].

In this work, we focus on the role of the QCD phase transition on the evolution of the young universe, with particular reference to the effect of the former on the evolution of the energy density fluctuations. We also show preliminary results obtained considering the effect of a primordial strong magnetic background on the aforementioned fluctuations. The primordial QCD phase transition is interesting because at that time a big change of the number of degree of freedom took place. The role of this primordial transition is still matter of debate. For example, in [2] it was realized that an inhomogeneous distribution of baryons due to a first-order QCD transition might change the primordial abundances of the light elements [11].

\section{EQUATIONS OF STATE FOR STRONGLY INTERACTING MATTER}

In this work we describe the plasma in the early universe as a quark-gluon plasma plus electroweak matter in thermal equilibrium. The energy density (as well as other thermodynamical quantities) are then written as

$$
\varepsilon=\varepsilon_{e w}+\varepsilon_{q g p},
$$

where $\varepsilon_{e w}, \varepsilon_{q g p}$ correspond to the energy densities of the electroweak and QCD plasmas respectively. The electroweak sector will be considered as a perfect gas of massless particles in thermal equilibrium at the temperature $T$, hence

$$
\varepsilon_{e w}=g_{e w} \frac{\pi^{2}}{30} T^{4}, \quad P_{e w}=g_{e w} \frac{\pi^{2}}{90} T^{4},
$$

with $g_{e w}=14.45$ [1]. On the other hand, for the QCD plasma we cannot ignore the strong interactions which in particular are very important in proximity of the phase transition. Therefore we need to use the appropriate equation of state for this matter. 

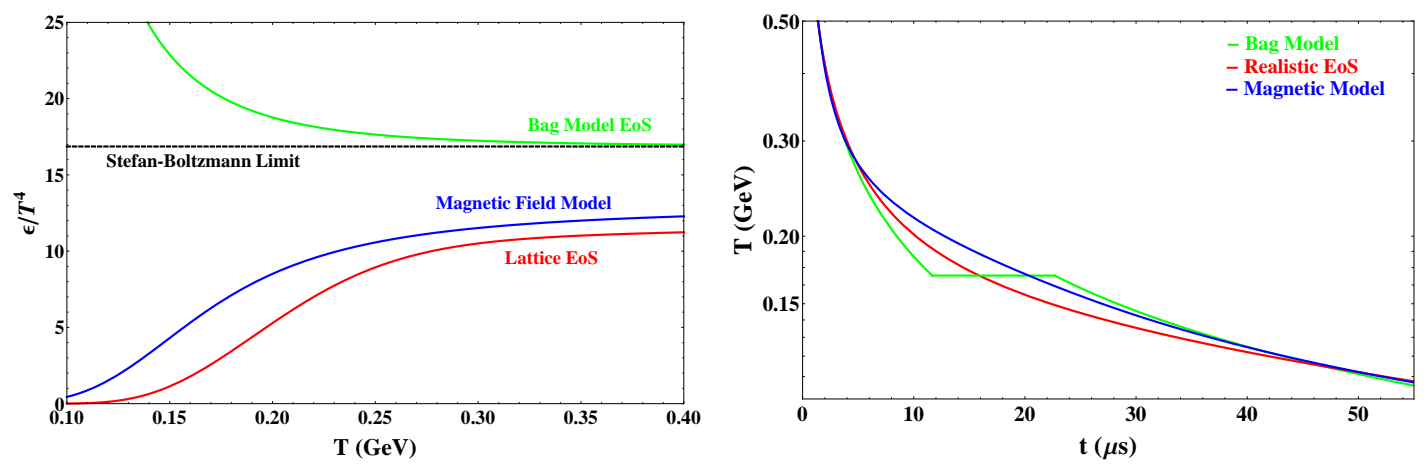

FIGURE 1. Left panel. Energy density of the QCD plasma against temperature. Red line represents the lattice EoS, the blue line the same realistic EoS with the inclusion of a strong primordial magnetic field, and the green line corresponds to the bag model EoS. For reference the Stefan-Boltzmann limit is reported with the black dashed line. Right panel. Temperature as a function of time for the three EoS discussed in the main text. Color convention is the same of the left panel.

The simplest way to take into account the properties of the QGP is the EoS of the MIT bag Model [8]. In the bag model one describes the short-distance dynamics by a collisionless gas of massless quarks and gluons, while the longdistance confinement effects are taken into account by subtracting a positive contribution to the pressure named the bag constant $B$. We present one result obtained within the bag model in this Section, for a standard value of the Bag constant $B=235 \mathrm{MeV}^{4}$, which is shown only for a matter of comparison with the more realistic cases discussed below.

As an example of realistic EoS for the QCD plasma we use the one recently computed on the lattice for the case of 2+1 flavor QCD [5]. Moreover, during the QCD transition strong magnetic fields might have been present [6, 15]. In order to take into account this possibility we present our preliminary results for the energy density fluctuations in presence of a strong magnetic background, using an effective model to compute the relevant equation of state [10]. Here we consider an admittedly large value of the magnetic field, $B \approx 10^{15}$ Tesla; this value of $B$ is chosen to estimate the maximum effect a magnetic field can have on the evolution of the energy density fluctuations in the early universe. The use of an effective model to describe the QCD plasma in presence of a magnetic background might appear opinable, since such model fails to reproduce the dependence of the chiral critical temperature on the magnetic field strength [3] (see however [7] and also the discussions in [12]). However our main point is to discuss the effect of the strengthening of the phase transition with the strength of $B$, a characteristic which is observed both in lattice simulations and in the model calculation, which is not related to the dependence of the critical temperature on $B$.

In Fig. 1 we plot $\varepsilon_{q g p}$ as a function of temperature. The black dashed line corresponds to the case of ideal gas of massless particles (the Stefan-Boltzmann limit) that is directly correlated to the number of degrees of freedom of the system. Red solid line corresponds to the case of the lattice QCD EoS; blue line to the EoS with magnetic field. For a matter of comparison we also show the case of the bag model by the solid green line. We remind that even in the most recent textbooks the temperature evolution of the Early Universe is usually discussed with a simple Bag model. The difference between the realistic EoS and the Stefan-Boltzmann limit indicates that the interactions in the plasma are non-negligible at temperatures which are relevant in our study temperatures.

\section{TEMPERATURE EVOLUTION}

In this Section we show our results for the temperature evolution in the early universe. To determine this quantity we solve the Friedmann equation that, assuming that the expansion of the Universe is isentropic, takes the form

$$
\frac{d \varepsilon}{d t}=-3 \sqrt{\frac{8 \pi G \varepsilon}{3}}(\varepsilon+P)
$$

The numerical solutions for the equations of state described in the previous Section are shown in the right panel of Fig. 1 that clearly shows how changing the equation of state affects the behaviour of temperature against time. The Bag Model (green line) presents a plateau during the transition corresponding to the critical temperature of about 170 $\mathrm{MeV}$, at which the phase transition is of first order. On the other hand because the QCD phase transition is actually a crossover, the use of the lattice EoS (red line) results in a smooth change of the temperature as a function of time. 


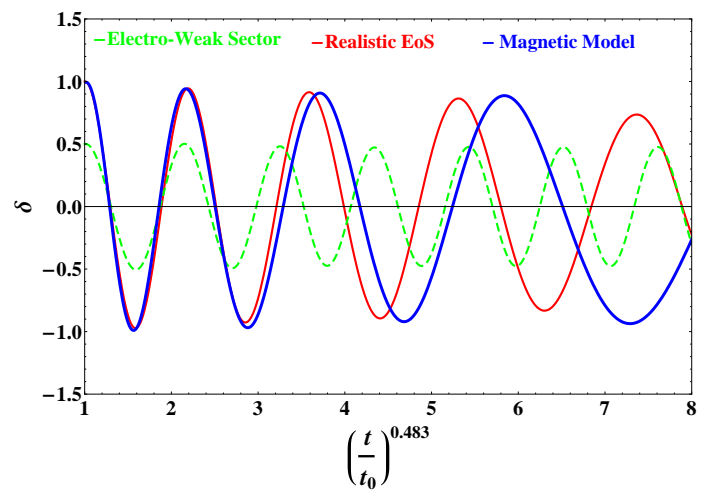

FIGURE 2. Time evolution of the energy density function with respect to the electro-weak part (green dashed line). The red line refers to the lattice QCD data while the blue line indicates the case in which a primordial magnetic field is included.

The magnetic field does not change the nature of the phase transition (blue line). We stress that the difference between the three cases are strictly confined to the time interval in which the transition takes place, while at later times the temperatures for the three different cases are very similar.

\section{ENERGY DENSITY FLUCTUATIONS}

The main goal of this work is to study the role of the primordial QCD transition on the energy density fluctuations in the early universe. If the QCD phase transition was of the first order then the speed of sound would vanish at the critical temperature, implying large oscillations of the energy density fluctuation. For the realistic case of the crossover we do expect the fluctuations to be damped because the speed of sound never reaches zero but simply decreases to a finite value in the crossover temperature range; nevertheless it is interesting to analyse how these fluctuations develop in presence of the crossover [13]. In order to accomplish this goal we solve the following system of equations [14]:

$$
\begin{gathered}
\frac{1}{H} \delta^{\prime}+3\left(c_{s}^{2}-w\right) \delta=\frac{k}{H} \psi-3(1+w) \alpha \\
\frac{1}{H} \psi^{\prime}+(1-3 w) \psi=-c_{s}^{2} \frac{k}{H} \delta-(1+w) \frac{k}{H} \alpha \\
\frac{1}{H} \delta_{e w}^{\prime}=\frac{k}{H} \psi_{e w}-4 \alpha \\
\frac{1}{H} \psi_{e w}^{\prime}=-\frac{k}{3 H} \delta_{e w}-\frac{4 k}{3 H} \alpha \\
{\left[\left(\frac{k}{H}\right)^{2}+\frac{9}{2}\left(1+w_{R}\right)\right] \alpha=-\frac{3}{2}\left(1+3 c_{s R}^{2}\right) \delta_{R},}
\end{gathered}
$$

where the prime denotes the derivative with respect to the conformal time $\eta$. In the above equations the most important quantity for our investivation is the function $\delta \equiv \delta \varepsilon / \varepsilon$, where $\delta \varepsilon$ correspond to the energy density fluctuation and $\varepsilon$ to the background energy density. Among the other variables involved in the dynamics, $\psi$ is related to the fluid velocities and $\alpha$ to the fluctuation of the temporal part of the metric tensor. The function $w$ is an input and corresponds the ratio background pressure over background energy density, which we compute case by case according to the input equation of state presented before. In the system of Eqs. (4)-(8) the first two equations describe the dynamics of the QCD plasma and follow from the energy-momentum conservation; the second couple of equations refer to the electro-weak sector and follow from the Euler equation of general relativity; finally the the last one follows from the Einstein $R_{0}^{0}$-equation and couples the QCD plasma to the electroweak one. For more details we refer to [13, 9].

In Fig. 2 we plot the result of our numerical solution of the system of Eqs. (4)-(8), focusing in particular on the energy density fluctuations $\delta$. The initial time is $t_{0}=1.35 \mu \mathrm{s}$ and is chosen in order to have an initial temperature 
of $500 \mathrm{MeV}$, while the final time corresponds to $110 \mu$ s which is well beyond the QCD transition. In the figure the green dashed line corresponds to the electro-weak component, while red (blue) solid line corresponds to the QCD EoS without (with) magnetic field. It is evident that the presence of the QCD crossover in the EoS at $B=0$ damps the energy density fluctuations in the considered time range in both cases, affecting also the frequency of these fluctuations. Moreover, effect of the magnetic field is to make this damping less efficient; this was expected because $B \neq 0$ makes the crossover a stiffer one, thus reducing the speed of sound in the crossover region compared to the speed of sound at $B=0$, and favouring the energy density fluctuations bringing the system behaviour closer to the one expected for a first order phase transition.

\section{CONCLUSIONS}

In conclusion, in this work we studied the effect of an equation of state which takes into account the existence of the QCD crossover on the evolution of the early universe. In particular we computed the time evolution of temperature and of energy density fluctuations by using as input the lattice QCD equation of state, describing the primordial QCD plasma at zero external fields, and a model equation of state which permits to describe the QCD plasma in presence of a strong magnetic background.

We found that the oscillations of the energy density fluctuations for the two aforementioned cases are damped during the QCD crossover, this implies that there are small chances of inhomogeneities phenomena during the Big Bang nucleosynthesis. When a strong magnetic background is added the damping is less effective, because in the latter case the QCD crossover is stiffer and makes the confinement closer to a first order phase transition, with a smaller speed of sound in the critical temperature range. As an extension of this work it will be interesting to use a lattice equation of state with magnetic field to study our problem, like the one computed recently in [4]. Studies along this line will be reported elsewhere.

\section{ACKNOWLEDGMENTS}

G. L. G. and V. G. acknowledge the ERC-STG funding under the QGPDyn grant.

\section{REFERENCES}

1. Quark-Gluon Plasma. Cambridge University Press, Cambridge, 2005.

2. J.H. Applegate, C.J. Hogan, and R.J. Scherrer. Cosmological quantum chromodynamics, neutron diffusion, and the production of primordial heavy elements. Ap.J., 329:572-579, 1988.

3. G.S. Bali, F. Bruckmann, G. Endrodi, Z. Fodor, S.D. Katz, et al. The QCD phase diagram for external magnetic fields. JHEP, 1202:044, 2012.

4. Claudio Bonati, Massimo D’Elia, Marco Mariti, Francesco Negro, and Francesco Sanfilippo. Equation of state and magnetic susceptibility of $N_{f}=2+1$ QCD with physical quark masses. 2013.

5. Szabolcs Borsanyi, Gergely Endrodi, Zoltan Fodor, Christian Hoelbling, Sandor Katz, et al. Transition temperature and the equation of state from lattice QCD, Wuppertal-Budapest results. J.Phys., G38:124101, 2011.

6. Leonardo Campanelli. Origin of Cosmic Magnetic Fields. Phys.Rev.Lett., 111:061301, 2013.

7. Jingyi Chao, Pengcheng Chu, and Mei Huang. Inverse magnetic catalysis induced by sphalerons. Phys.Rev., D88:054009, 2013.

8. A. Chodos, R.L. Jaffe, K. Johnson, and C.B Thorn. Baryon structure in the bag theory. Phys.Rev., D10:2599-2604, 1974.

9. Wojciech Florkowski. The realistic QCD equation of state in relativistic heavy-ion collisions and the early Universe. Nucl.Phys., A853:173-188, 2011.

10. Raoul Gatto and Marco Ruggieri. Deconfinement and Chiral Symmetry Restoration in a Strong Magnetic Background. Phys.Rev., D83:034016, 2011.

11. Toshitaka Kajino. Inhomogeneous big-bang model, revived, and evolution of the light elements in cosmic rays. Nucl.Phys., A588:339c-344c, 1995.

12. Toru Kojo and Nan Su. The quark mass gap in a magnetic field. Phys.Lett., B720:192-197, 2013.

13. Christoph Schmid, Dominik J. Schwarz, and Peter Widerin. Peaks above the Harrison-Zel'dovich spectrum due to the quark gluon to hadron transition. Phys.Rev.Lett., 78:791-794, 1997.

14. Christoph Schmid, Dominik J. Schwarz, and Peter Widerin. Amplification of cosmological inhomogeneities from the QCD transition. Phys.Rev., D59:043517, 1999.

15. T. Vachaspati. Magnetic fields from cosmological phase transitions. Phys.Lett., B265:258-261, 1991. 